\title{
Validation of a prospective urinalysis-based prediction model for ICU-resources and outcome of Covid-19 disease: A multicenter cohort study
}

Oliver Gross ( $\square$ gross.oliver@med.uni-goettingen.de )

University Medicine Goettingen

Onnen Moerer

University Medical Center Göttingen

Thomas Rauen

RWTH Aachen University

Jan Böckhaus

University Medical Center Göttingen

\section{Elion Hoxha}

University Medical Center Hamburg-Eppendorf

Achim Jörres

University Witten/Herdecke Medical Center Cologne-Merheim

Matthias Kamm

University Witten/Herdecke Medical Center Cologne-Merheim

Amin Elfanish

University Witten/Herdecke Medical Center Cologne-Merheim

Wolfram Windisch

University Witten/Herdecke

Michael Dreher

Medical Clinic V, RWTH Aachen University

Juergen Floege

RWTH Aachen University

Stefan Kluge

University Medical Center Hamburg-Eppendorf

Christian Schmidt-Lauber

University Medical Center Hamburg-Eppendorf

Jan-Eric Turner

University Medical Center Hamburg-Eppendorf

\section{Samuel Huber}

University Medical Center Hamburg-Eppendorf

\section{Marylyn M. Addo}


University Medical Center Hamburg-Eppendorf

\section{Simone Scheithauer}

University Medical Center Göttingen (UMG), Georg-August University

\section{Tim Friede}

University Medical Center Göttingen

\section{Gerald S. Braun}

RWTH Aachen University

\section{Tobias B. Huber}

University Medical Center Hamburg-Eppendorf

\section{Sabine Blaschke}

University Medical Center Göttingen

\section{Research Article}

Keywords: Covid-19, ICU-resources, risk stratification, clinical outcomes, preventive measures

Posted Date: October 23rd, 2020

DOI: https://doi.org/10.21203/rs.3.rs-97097/v1

License: (c) (1) This work is licensed under a Creative Commons Attribution 4.0 International License. Read Full License 
Validation of a prospective urinalysis-based prediction model for ICU-resources and outcome of Covid-19 disease: A multicenter cohort study

Oliver Gross, ${ }^{1}$ Onnen Moerer, ${ }^{2 \#}$ Thomas Rauen, ${ }^{3 \#}$ Jan Böckhaus, ${ }^{1 \#}$ Elion Hoxha, ${ }^{4 \#}$ Achim Jörres, ${ }^{5}$ Matthias Kamm, ${ }^{5}$ Amin Elfanish, ${ }^{5}$ Wolfram Windisch, ${ }^{6}$ Michael Dreher, ${ }^{7 \S}$ Juergen Floege,${ }^{3}$ Stefan Kluge,${ }^{8}$ Christian Schmidt-Lauber, ${ }^{4}$ Jan-Eric Turner, ${ }^{4}$ Samuel Huber, ${ }^{9}$ Marylyn M. Addo, ${ }^{9}$ Simone Scheithauer, ${ }^{10}$ Tim Friede, ${ }^{11 *}$ Gerald S. Braun, ${ }^{3 *}$ Tobias B. Huber,${ }^{4 *}$ Sabine Blaschke, ${ }^{12 *}$

\# contributed equally (first authorship);

* contributed equally (senior authorship);

$\S$ for the COVAS registry

${ }^{1}$ Clinic for Nephrology and Rheumatology, University Medical Center Göttingen, Göttingen. Germany

${ }^{2}$ Clinic of Anaesthesiology, University Medical Center Göttingen, Göttingen, Germany

${ }^{3}$ Division of Nephrology and Clinical Immunology, RWTH Aachen University, Aachen, Germany

${ }^{4}$ III. Department of Medicine, University Medical Center Hamburg-Eppendorf, Hamburg, Germany

${ }^{5}$ Department of Medicine I, Nephrology, Transplantation and Medical Intensive Care, University Witten/Herdecke Medical Center Cologne-Merheim, Cologne, Germany

${ }^{6}$ Department of Pneumology and Critical Care Medicine, University Witten/Herdecke, Medical Center Cologne-Merheim, Cologne, Germany

${ }^{7}$ Department of Pneumology and Intensive Care Medicine, Medical Clinic V, RWTH Aachen University, Aachen, Germany

${ }^{8}$ Department of Intensive Care, University Medical Center Hamburg-Eppendorf, Hamburg, Germany

${ }^{9}$ I. Department of Medicine, University Medical Center Hamburg-Eppendorf, Hamburg, Germany

${ }^{10}$ Institute of Infection Control and Infectious Diseases, University Medical Center Göttingen (UMG), Georg-August University Göttingen, Germany

${ }^{11}$ Department of Medical Statistics, University Medical Center Göttingen, Göttingen, Germany

${ }^{12}$ Emergency Department, University Medical Center Göttingen, Göttingen, Germany

\section{Corresponding author:}

Oliver Gross, M.D.

Clinic for Nephrology and Rheumatology

University Medical Center Göttingen

Robert-Koch Str. 40; 37075 Goettingen, Germany

tel: +49551398912 fax: +49551398906

email: gross.oliver@med.uni-goettingen.de 


\section{Abstract}

Purpose: Identifying preventive strategies in Covid-19 patients helps to improve ICU-resourceallocation and reduce mortality. We recently demonstrated in a post-mortem cohort that SARSCoV-2 renal tropism was associated with kidney injury, disease severity and mortality. We also proposed an algorithm to predict the need for ICU-resources and the risk of adverse outcomes in Covid-19 patients harnessing urinalysis and protein/coagulation parameters on admission for signs of kidney injury. Here, we aimed to validate this hypothesis in a multicenter cohort.

Methods: Patients hospitalized for Covid-19 at four tertiary centers were screened for an available urinalysis, serum albumin (SA) and antithrombin-III activity (AT-III) obtained prospectively within $48 \mathrm{~h}$ upon admission. The respective presumed risk for an unfavorable course was categorized as "low", "intermediate" or "high", depending on a normal urinalysis, an abnormal urinalysis with $S A \geq 2 \mathrm{~g} / \mathrm{dl}$ and $\mathrm{AT}-\mathrm{III} \geq 70 \%$, or an abnormal urinalysis with at least one SA or AT-III abnormality. Time to ICU or death within ten days served as primary, in-hospital mortality and required organ support served as secondary endpoints.

Results: Among a total of $\mathrm{N}=223$ screened patients, $\mathrm{N}=145$ were eligible for enrollment, falling into the low $(\mathrm{N}=43)$, intermediate $(\mathrm{N}=84)$, and high risk $(\mathrm{N}=18)$ categories. The risk for ICU transfer or death was $100 \%$ in the high risk group and significantly elevated in the composite of high and intermediate risk as compared to the low risk group (63.7\% vs. $27.9 \%$; HR 2.6; 95\%-Cl 1.4 to $4.9 ; \mathrm{P}=0.0020)$. Having an abnormal urinalysis was associated with mortality, need for mechanical ventilation, extra-corporeal membrane oxygenation (ECMO) or renal replacement therapy (RRT).

Conclusion: Our data confirm that Covid-19-associated urine abnormalities on admission predict disease aggravation and need for ICU. By engaging a simple urine dipstick on hospital admission our algorithm allows for early preventive measures and appropriate patient stratification. (ClinicalTrials.gov number NCT04347824) 


\section{Keywords}

Covid-19, ICU-resources, risk stratification, clinical outcomes, preventive measures.

\section{Take home message}

Our study validated a prediction model to foresee multiorgan disease severity and to stratify preventive measures in Covid-19 patients on admission to hospital. This provides a strategic advantage when it comes to the triage and surveillance of Covid-19 patients seeking hospital treatment since the algorithm helps health care workers to anticipate need for ICU, mechanical ventilation, ECMO and renal replacement therapy and to treat or even prevent impending complications such as thromboembolism or fluid-overload. 


\section{Introduction}

Coronavirus 2019 disease (Covid-19) is initiated by infection of the upper respiratory tract with the severe acute respiratory syndrome coronavirus 2 (SARS-CoV-2) [1, 2]. Currently known major mechanisms of the disease comprise viral replication in multiple organs including the kidney and involving multiple cell types, vascular endothelitis, thrombosis, and systemic cytokine storm [3-9]. While most patients experience mild to moderate disease amenable to ambulatory management, a subgroup requires hospitalization. A proportion of this latter group will exhibit a further decline to multiorgan failure requiring mechanical ventilation, extra corporeal membrane oxygenation (ECMO) or renal replacement therapy (RRT) yielding a high mortality [10-17]. Means assisting in the early clinical identification of such patients would bare the potential of improving allocation of medical resources and thus outcome [18]. In a previous observational pilot study in our emergency department we found that early on, abnormalities of the urine, serum albumin and antithrombin-III (AT-III) were associated with worse outcomes of Covid-19. Consequently, we proposed the hypothesis in the Lancet that these markers may serve indicate kidney involvement and loss of important proteins to construct a prediction tool for COVID-19 severity [19]. Here we present the multicenter cohort validation study (NCT04347824) of this hypothesis.

The rationale for employing urinalysis is further supported by our recent collaborative autopsy study demonstrating that renal tropism of SARS-CoV-2 was associated with overall disease severity including premature death and with the development of acute kidney injury (AKI) [20]. Tubular, glomerulo-endothelial and interstitial inflammatory damage may all be reflected in the urine in form of low osmolarity, albuminuria/hematuria and leukocyturia, respectively. In addition, it is currently believed that an abnormal urinalysis can also reflect non-viral changes induced by systemic Covid-19 [21]. For practical reasons, here we define both etiologies of urinary abnormalities as 'Covid-19 associated kidney injury'. Indeed, urinary abnormalities such as protein loss may both indicate and drive severe disease. 
The rationale for adding serum albumin and AT-III activity to the novel algorithm derive from our pilot study's observation that they were particularly low among the sickest Covid-19 patients and that the administration of these substances for substitution in the ICU setting surged during the first Covid-19 wave in Germany [19]. Both parameters are widely used by emergency and intensive care physicians as they play a major role in the pathogenesis of sepsis [22], and also by nephrologists in nephrotic patients in whom these two proteins are lost through an insufficient glomerular filter [23]. Low serum albumin induces fluid overload, pulmonary edema and circulatory failure, all of which are known to be major cause of death in Covid-19 multiorgan involvement [3-9]. Low AT-III activity triggers thromboembolic events and counteracts the beneficial effect of heparin. 


\section{Methods}

Data quality, quality assurance and study population

Starting in April 2020, in this multi-center cohort study all adult patients hospitalized to four German tertiary medical centers were screened for the key inclusion criteria: (1) approved SARS-CoV-2 diagnosis (by PCR), (2) urinary status during hospital stay, and (3) the patient's written consent. Among all screened patients, we included patients with an urinalysis obtained prospectively within $48 \mathrm{~h}$ upon hospital admission into the present validation analysis.

Data collection was performed using a standardized, ICH-GCP-conform and pseudonymized questionnaire assessing age, sex, weight, height, information on Covid-19 diagnosis as well as information on chronic diseases, urinary status, serum albumin, AT-III activity, need for ICU transfer, need for and time on mechanical ventilation, ECMO, RRT and death as available from routine clinical records. According to the German Medicines Act, the study was approved by the leading institutional review board (IRB) of the UMG Göttingen $(41 / 4 / 20)$, and all others. All co-authors collected the data and vouch for the completeness and accuracy of the data and analyses and for the fidelity of the study to the protocol. The decision to submit the manuscript for publication was made by all the authors. Exchange of pseudonymized data was secured by individual contracts (data processing and cooperation agreements) between the participating centers. To maintain high-standard data quality and to reduce selection bias, data were only analyzed after the SAP (statistical analysis plan) was completed and database closure. "Transparent reporting of a multivariable prediction model for individual prognosis" (TRIPOD) criteria were entirely applied (see TRIPOD Checklist in the Suppl. material) as well as the STROBE Statement checklist for cohort studies. The trial was registered prospectively at www.ClinicalTrials.gov (NCT04347824).

\section{Urinalysis and patient stratification}

An abnormal urinary status was defined as either anuria or as at least two of the following criteria: (1) urine osmolarity (or urine specific gravity, depending on the local preference) below normal values; (2) leukocyturia; (3) hematuria; (4) albuminuria / proteinuria. If urine was positive 
for nitrite or bacteria, at least three of these criteria were necessary to define an abnormal urinary status.

Serum albumin and AT-III activity were assessed by routine laboratory tests. Eligible patients were then stratified into three predefined groups as follows: (1) low-risk for Covid-19 kidney injury ("green"): patients without urinary abnormalities; (2) intermediate-risk ("yellow"): patients with an abnormal urinary status, serum albumin $\geq 2.0 \mathrm{~g} / \mathrm{dl}$ and AT-III activity $\geq 70 \%$; (3) high-risk ("red"): patients with an abnormal urinary status, serum albumin $<2.0 \mathrm{~g} / \mathrm{dl}$ or AT-III activity $<70 \%$.

\section{Study endpoints (for full study protocol, see supplemental material)}

The primary endpoint was time to event (in days) defined as time to ICU transfer or death, whatever occurred first, within the first ten days upon hospital admission. Secondary endpoints included the number of patients who died, were transferred to an ICU or required mechanical ventilation, ECMO or RRT. Additionally, we captured the lowest available serum albumin and AT-III activity levels.

\section{Statistical analyses (for full statistical analysis plan, see supplemental material)}

As per study protocol, the aim was to recruit at least 100 patients and at most 250 patients into the study. The sample size was based on feasibility. The primary comparison is between low-risk and intermediate / high-risk patients with regard to the primary endpoint, i.e. time to ICU transfer or death. The expectation was that only $20 \%$ of patients will be in the low-risk group whereas $80 \%$ will be in the intermediate or high-risk groups. The risk for a primary outcome event was considered to be $\sim 25 \%$ (at day 10 ) in the low-risk group and $\sim 50 \%$ in the composite intermediate / high-risk group with a relative risk (RR) of 2 . Under these assumptions a total sample size of 183 or 240 patients yields a power of $80 \%$ or $90 \%$, respectively, at the typical two-sided significance level of $5 \%$. When postulating a risk of $30 \%$ in the low-risk group and otherwise the same assumptions (in particular $R R=2$ ), a total sample size of 130 (172) patients yields a power of $80 \%(90 \%)$. 
Baseline demographics and clinical characteristics are summarized using appropriate statistics. The distributions of continuous variables are also displayed as box plots. The baseline characteristics are presented for the whole study population as well as stratified by the three risk groups. Here both analysis sets are considered, for the association with disease severity (crosssectional study) and for the prediction of disease aggravation.

To assess the association of the risk groups as determined by the novel algorithm [19], a frequency table of the risk groups and the type of ward are provided. The association is formally tested by a chi-square test. A two-sided p-value is reported.

For prediction of disease aggravation, the primary endpoint time from hospital admission to transfer to ICU or death (whatever comes first) was analyzed using a Cox proportional hazards model with the risk groups as the independent variable. The primary null hypothesis was that the hazard ratio $(\mathrm{HR})$ for low-risk vs. intermediate / high-risk is higher or equal to one, i.e. H0: $H R \geq 1$. The hazard ratio is reported with $95 \%$ confidence interval $(\mathrm{Cl})$ and $\mathrm{P}$-value (Wald-type test). The performance of the prognostic model is evaluated using the $\mathrm{C}$-index and calibration slope (reported with standard errors (SE)). The data are visualized by Kaplan-Meier curves stratified by risk group.

In supporting analyses, additional prognostic variables are added to the models. These include age, sex, presence of any comorbidity (e.g. kidney function, diabetes, cardiovascular disease, chronic respiratory disease). 


\section{Results}

\section{Study population}

Among the entire cohort of 223 patients hospitalized for Covid-19, 145 individuals had an urinalysis prospectively obtained within $48 \mathrm{~h}$ upon hospital admission and thereby qualified for inclusion into the validation analysis, whereas 78 patients had an urinalysis that was obtained later during hospital stay $(\mathrm{N}=64)$ or were secondary referrals from ICU of other medical centers $(\mathrm{N}=14$; Figure 1).

When looking at the entire cohort $(\mathrm{N}=223 ; \mathrm{N}=58,26 \%$ with normal; $\mathrm{N}=165,74 \%$ with abnormal urinalysis at some stage during Covid-19) we found similar baseline characteristics of both groups and could confirm the overall association of urine abnormalities with dismal outcomes in a statistically significant fashion (see Suppl. Table 1 for details).

\section{Validation of the Prediction Model}

Based on the proposed algorithm derived from our pilot study in Covid-19 patients (Gross et al, Lancet 2020) [19], our major goal was to validate the predictive value of this algorithm in those 145 Covid-19 patients who had an urinalysis obtained within 48h upon hospital admission. Based on our pre-specified diagnostic classification criteria (see methods), 43 individuals (30\%) had a normal (categorized to "green", low-risk) and $102(70 \%)$ had an abnormal urinalysis with the latter group sub-differentiating into 84 intermediate-risk ("yellow") and 18 high-risk ("red") patients (Figure 1, Table 1). Patients with an abnormal urinalysis at hospitalization had a higher risk for ICU transfer or death within ten days upon hospitalization $(\mathrm{N}=65 / 102,63.7 \%)$ as compared to those without urinary abnormalities $(\mathrm{N}=12 / 43,27.9 \%$; HR 2.6; $95 \%-\mathrm{Cl} 1.4$ to 4.9; $\mathrm{P}=0.0020 ;$ Tables 2/3, Suppl. Figure 1). The HR remained stable even after adjusting for age, sex and co-morbidities (Suppl. Table 2). Based on the risk stratification, all patients in the high-risk group, $51.2 \%$ of the intermediate-risk group and only $23.3 \%$ of the low-risk group reached the primary composite endpoint (Figure 2, Table 2). The C-index (SE) for this simple Cox regression was $0.61(0.03)$. The adjustment of the prediction model for age, sex and co-morbidities revealed a robust hazard ratio for Covid-19 kidney injury, which was 
consistent with the unadjusted hazard ratio (C-index $0.68(0.04))$ (Suppl. Table 2). Patients with urinary abnormalities on admission to hospital had a higher in-hospital mortality $(29.4 \%$ vs. $11.6 \%$ ), a higher risk to need mechanical ventilation (44.1\% vs. $14.0 \%)$, ECMO therapy (10.8\% vs. $2.3 \%)$ and RRT (30.7\% vs. $11.6 \%$; Table 2). Primary and secondary outcome events were particularly elevated in high-risk patients as compared to those at intermediate risk (Table 2, Figure 2). In parallel, despite death as a competing event, the risk groups also correlated with the average patient-time on ICU and for days on mechanical ventilation, ECMO-therapy, and RRT (Table 2), which might be of special interest in scenarios of limited resources and limited ICU capacities [10].

\section{Serum albumin and AT-III activity in the prediction cohort}

The prospectively chosen categorical ranges for serum albumin and AT-III served to substratify the intermediate and high-risk groups as stated above in the validation of the prediction model. Next, we sought to get further insight on their association as indicators or hypothetic drivers of Covid-19. To this end we plotted the earliest available and lowest serum albumin and AT-III levels of the three risk groups against each other (Figure 3). Median values of these parameters declined with the traffic light coding system from "green" to "red", thereby supporting the definitions used. The delta between earliest and lowest value was surprisingly stark in the intermediate-risk group for serum albumin and in the high-risk-group for AT-III activity. This might reflect the dynamics of the disease within these categories. While only $3.0 \%$ of low-risk patients had a minimal serum albumin below $2.0 \mathrm{~g} / \mathrm{dl}$, this was noted in $30.0 \%$ and $94.4 \%$ of the intermediate- and high-risk groups, respectively. Similarly, $22.7 \%$ of the low-risk, $40.4 \%$ of the intermediate-risk and $66.7 \%$ of the high-risk group reached minimum levels of AT-III lower than $70 \%$. This translates to almost $50 \%$ of intermediate-risk patients deteriorating from "yellow" to the "red" category during their hospital stay, thus implying a dynamic change in disease severity. Although outside of the scope of the present study, we hypothesize that these parameters could help to predict risks for the further course of the disease, opening up a chance to intervene and prevent complications. 


\section{Discussion}

The present validation study confirmed that a composite and easily accessible algorithm involving an urinalysis, serum albumin and AT-III activity at hospitalization is suited to predict the severity and the further course of Covid-19 disease, which also includes the need for ICU resources.

The majority of patients at intermediate risk were the most common group. Notably, $30 \%$ of this group showed a decline in their serum albumin to the high-risk category (below $2.0 \mathrm{~g} / \mathrm{dl}$ ) during their hospital stay: AT-III activity fell in $40 \%$ of the intermediate-risk patients below the critical cut-off of $70 \%$ (Figure 3). Low AT-III activity is known to trigger thromboembolic complications. Preventive measures require a much more aggressive dosing of heparin, substitution of AT-III, or the therapeutic switch to another anticoagulant that does not work via AT-III. Our algorithm indicates that those with normal urine have a substantially lower risk progressing only in $25 \%$ of cases to ICU or death. Yet, the algorithm puts a high number of patients in the focus: this group might benefit most from our algorithm as patients at intermediate risk are put in the focus of preventive measures earlier.

The present data do not allow for a conclusive statement, as to which pathomechanisms contribute most to the low albumin and low AT-III activity. One may speculate that low AT-III activity is caused by increased consumption due to hypercoagulable state, increased loss by capillary leak, decreased hepatic production and, importantly, renal loss. Covid-19 patients exhibit frequent thromboembolic events, and pulmonary embolism has evolved as major killer for inpatients and outpatients with Covid-19 [24-27]. Low levels of AT-III trigger this complication and counteract the effect of the most common anticoagulant heparin. In addition, patients with Covid-19 show pulmonary interstitial edema due to severe fluid overload, similar to nephrotic syndrome, and circulatory failure requiring high doses of catecholamines [22]. In the latter setting, low serum albumin triggers this complication and is believed to be the result of increased loss by capillary leak and decreased hepatic production. Patients with Covid-19 show 
circulatory failure and require high doses of catecholamines. Low serum albumin may also explain reduced response to some medications by impaired plasma protein binding.

\section{Limitations}

Our study has several strengths and limitations. A first limitation relates to the use of noninterventional data from clinical routine that has also led to the loss of data points in a number of patients and the partial use of retrospective data, which can be explained by the extreme circumstances of a pandemic [28]. However, the overall study design has a clear prospective nature, as our key inclusion criteria is the predictive urinalysis, which had to be obtained on hospital admission within 48 hours and on the same day or prior the event (i.e. primary endpoint). Notably, median serum-creatinine on hospital admission (Table 1) did not differ in between the low-risk group and the intermediate/high-risk group, which clearly underlines the value of the urinalysis for our prediction model. As the second limitation, we acknowledge that it is currently impossible to discriminate from urine alone between SARS-CoV-2 infection of the kidney, by ICU-acute kidney injury, cytokine storm or additional confounders [29]. However, discrimination between acute or chronic renal injury might be less important in the triage room of the emergency department, when the highest need of the Covid-19 patient is a fast and easy to use risk-assessment [30]. As the third limitation, additional diseases including CKD might be confounding factors for need for ICU and mortality. Ten percent of patients worldwide have preexisting CKD, and urinary tract infections or indwelling catheters represent a potential confounder, presumably in those patients with an urinalysis later than $48 \mathrm{~h}$ after admission to hospital. Lastly, a limitation lies in the early recordings of albumin and ATIII on hospital admission whereas the disease is highly dynamic, thus inherently compromising on the predictive power as compared to repeated measurements. As one strength our study's C-index, which is indicative of the accuracy of a prediction model, was 0.61 . This value suggests our prediction model being as accurate and useful in the real-life setting, since it stayed significant despite potential negative influences such as screening failures due to the lack of urinalysis or the competing risks of death ICU transfer as primary endpoints. 


\section{Conclusions and clinical implications}

This simple algorithm may provide a strategic advantage when it comes to the triage and surveillance of Covid-19 patients seeking hospital treatment. The algorithm can also be used in a modified way for better surveillance of outpatients and more vulnerable populations such as elderly patients in nursing homes. We advocate its use serve therapeutic trials for better allocation of patients at risk or with the most need for specific therapies [31-35]. Furthermore, as

preventive measures will improve over time, the algorithm can be adapted with updated recommendations. 


\section{Conflicts of interest and funding}

\section{Role of the funding source}

In a peer-reviewed two-step application process, the UMG Göttingen applied for Government funding (Covid-19 program) by The German Federal Ministry of Education and Research. The application process did not have any influence on the trial design or analyses. In addition, this work was supported by the DFG (CRC1192 to JET, EH and TBH) and (GR 1852/6-1 to OG), and the BMBF (STOP-FSGS-01GM1518C to TBH). The corresponding author had full access to all the data in the study and had final responsibility for the decision to submit for publication.

\section{Authors' contributions, conflict of interest statements}

All co-authors collected the data and vouch for the completeness and accuracy of the data and analyses and for the fidelity of the study to the protocol. The decision to submit the manuscript for publication was made by all the authors. All authors report no conflict of interest in relation to this observational cohort-study.

\section{Data sharing statement}

The full trial protocol and the statistical analysis plan can be accessed as supplementary material. The full data set can be assessed on individual request.

\section{Acknowledgements}

We thank Tanja Albrecht-Nock and Ute Floege for deliberate data acquisition. 


\section{References}

1 Zhu N, Zhang D, Wang W, et al (2020) A novel coronavirus from patients with pneumonia in China, 2019. N Engl J Med 382: 727-33

2 Chen N, Zhou M, Dong X, et al (2020) Epidemiological and clinical characteristics of 99 cases of 2019 novel coronavirus pneumonia in Wuhan, China: a descriptive study. Lancet 395: 507-13

3 Puelles VG, Lütgehetmann M, Lindenmeyer MT, et al (2020) Multiorgan and Renal Tropism of SARSCoV-2. N Engl J Med 383: 590-592

4 Mehta P, McAuley DF, Brown M, Sanchez E, Tattersall RS, Manson JJ (2020) COVID-19: consider cytokine storm syndromes and immunosuppression. Lancet 395: 1033-34

5 Ackermann M, Verleden SE, Kuehnel M, et al (2020) Pulmonary Vascular Endothelialitis, Thrombosis, and Angiogenesis in Covid-19. N Engl J Med 383: 120-128

6 Liu PP, Blet A, Smyth D, Li H (2020) The science underlying COVID-19: implications for the cardiovascular system. Circulation 142: 68-78

7 Helms J, Tacquard C, Severac F, et al (2020) High risk of thrombosis in patients with severe SARSCoV-2 infection: a multicenter prospective cohort study. Intensive Care Med 46: 1089-98

8 Varga Z, Flammer AJ, Steiger P, et al (2020) Endothelial cell infection and endotheliitis in COVID-19. Lancet 395: 1417-8

9 Pei G, Zhang Z, Peng J, et al (2020) Renal involvement and early prognosis in patients with COVID-19 pneumonia. J Am Soc Nephrol 31: 1157-65

10 Emanuel EJ, Persad G, Upshur R, et al (2020) Fair Allocation of Scarce Medical Resources in the Time of Covid-19. N Engl J Med 382: 2049-55

11 Richardson S, Hirsch JS, Narasimhan M, et al (2020) Presenting characteristics, comorbidities, and outcomes among 5700 patients hospitalized with COVID-19 in the New York City area. JAMA 323: 2052-59

12 Zhou F, Yu T, Du R, et al (2020) Clinical course and risk factors for mortality of adult inpatients with COVID-19 in Wuhan, China: a retrospective cohort study. Lancet 395: 1054-62

13 Huang C, Wang Y, Li X, et al (2020) Clinical features of patients infected with 2019 novel coronavirus in Wuhan, China. Lancet 395: 497-506

14 Wu Z, McGoogan JM (2020) Characteristics of and important lessons from the coronavirus disease 2019 (COVID-19) outbreak in China: summary of a report of 72314 cases from the Chinese Center for Disease Control and Prevention. JAMA Feb 24. doi: 10.1001/jama.2020.2648. Online ahead of print

15 Goyal P, Choi JJ, Pinheiro LC, et al (2020) Clinical characteristics of Covid-19 in New York City. N Engl J Med 382: 2372-74

16 Guan W, Ni Z, Hu Y, et al (2020) Clinical characteristics of coronavirus disease 2019 in China. N Engl J Med 382: 1708-20

17 Wu C, Chen X, Cai Y, et al (2020) Risk factors associated with acute respiratory distress syndrome and death in patients with coronavirus disease 2019 pneumonia in Wuhan, China. JAMA Intern Med 180(7): $1-11$

18 Fagiuoli S, Lorini FL, Remuzzi G, Covid-19 Bergamo Hospital Crisis Unit (2020) Adaptations and Lessons in the Province of Bergamo. N Engl J Med 382: e71

19 Gross O, Moerer O, Weber M, et al (2020) COVID-19-associated nephritis: early warning for disease severity and complications? Lancet 395: e87-e88

20 Braun F, Lütgehetmann M, Pfefferle S, et al (2020) SARS-CoV-2 renal tropism associates with acute kidney injury. Lancet Aug 14: S0140-6736(20)31759-1. doi:10.1016/S0140-6736(20)31759-1. Online ahead of print. 
21 Batlle D, Soler MJ, Sparks MA, et al (2020) Acute Kidney Injury in COVID-19: Emerging Evidence of a Distinct Pathophysiology. J Am Soc Nephrol 31: 1380-1383

22 Sungurlu S, Kuppy J, Balk RA (2020) Role of Antithrombin III and Tissue Factor Pathway in the Pathogenesis of Sepsis. Crit Care Clin 36: 255-265

23 Noone DG, lijima K, Parekh R (2018) Idiopathic nephrotic syndrome in children. Lancet 392: 61-74

24 Wynants L, Van Calster B, Collins GS, et al (2020) Prediction models for diagnosis and prognosis of covid-19 infection: systematic review and critical appraisal. Version 2. BMJ Apr 7; 369: m1328. doi: 10.1136/bmj.m1328

25 Sun Q, Qiu H, Huang M, Yang Y (2020) Lower mortality of COVID-19 by early recognition and intervention: experience from Jiangsu Province. Ann Intensive Care 10: 33

26 Edler C, Schröder AS, Aepfelbacher M, et al (2020) Dying with SARS-CoV-2 infection-an autopsy study of the first consecutive 80 cases in Hamburg, Germany. Int J Legal Med 134: 1275-84

27 Tang N, Bai H, Chen X, Gong J, Li D, Sun Z (2020) Anticoagulant treatment is associated with decreased mortality in severe coronavirus disease 2019 patients with coagulopathy. J Thromb Haemost 18: 1094-9

28 Karagiannidis C, Mostert C, Hentschker C, Voshaar T, Malzahn J, et al (2020) Case characteristics, resource use, and outcomes of 10021 patients with COVID-19 admitted to 920 German hospitals: an observational study. Lancet Respir Med 8(9): 853-862

29 Vijayan A, Humphreys BD (2020) SARS-CoV-2 in the kidney: bystander or culprit? Nat Rev Nephrol 14: 1-2.

30 Seymour CW (2020) A cooperation of the doves. Intensive Care Med 18:1-2

31 Poston JT, Patel BK, Davis AM (2020) Management of critically ill adults with COVID-19. JAMA Mar 26. doi: $10.1001 /$ jama.2020.4914. Online ahead of print.

32 Cao B, Wang Y, Wen D, et al (2020) A trial of lopinavir-ritonavir in adults hospitalized with severe Covid-19. N Engl J Med 382: 1787-99

33 Wang Y, Zhang D, Guanhua D, et al (2020) Remdesivir in adults with severe Covid-19: a randomized, double-blind, placebo-controlled, multicentre trial. Lancet 395: 1569-78

34 RECOVERY Collaborative Group, Horby P, Lim WS, Emberson JR, et al (2020) Dexamethasone in Hospitalized Patients with Covid-19 - Preliminary Report. N Engl J Med Jul 17: NEJMoa2021436. doi: 10.1056/NEJMoa2021436. Online ahead of print

35 Duan K, Liu B, Li C, et al (2020) Effectiveness of convalescent plasma therapy in severe COVID-19 patients. Proc Natl Acad Sci U S A 117: 9490-6 


\begin{tabular}{|c|c|c|c|c|c|c|c|c|}
\hline \multirow{2}{*}{$\begin{array}{l}\text { Baseline characteristic } \\
\text { Male sex - no. (\%) }\end{array}$} & \multicolumn{2}{|c|}{ green $(\mathrm{N}=43)$} & \multicolumn{2}{|c|}{ yellow $(\mathrm{N}=84)$} & \multicolumn{2}{|c|}{$\operatorname{red}(\mathrm{N}=18)$} & \multicolumn{2}{|c|}{ non-green $(\mathrm{N}=102)$} \\
\hline & $29 / 43$ & $(67.4)$ & $53 / 84$ & $(63.1)$ & $12 / 18$ & $(66.7)$ & $65 / 102$ & $(63.7)$ \\
\hline Median serum-creatinine (IQR) - mg/dl & 0.97 & $(0.97-1.18)$ & & & & & 1.0 & $(0.76-1.63)$ \\
\hline Median age (IQR) - year & 61 & $(49-75)$ & 71 & $(60-79)$ & 67 & $(58-74)$ & 70 & $(59-78)$ \\
\hline 20 to 39 years of age & $8 / 43$ & $(18.6)$ & $4 / 84$ & $(4.8)$ & $0 / 18$ & (0) & $4 / 102$ & (3.9) \\
\hline 40 to 59 years of age & $12 / 43$ & $(27.9)$ & $17 / 84$ & $(20.2)$ & $5 / 18$ & $(27.8)$ & $22 / 102$ & $(21.6)$ \\
\hline 60 to 79 years of age & $20 / 43$ & $(46.5)$ & $44 / 84$ & $(52.4)$ & $11 / 18$ & $(61.1)$ & $55 / 102$ & $(53.9)$ \\
\hline 80 and older years of age & $3 / 43$ & (7) & $19 / 84$ & $(22.6)$ & $2 / 18$ & $(11.1)$ & $21 / 102$ & $(20.6)$ \\
\hline Median body mass index BMI (IQR) & 27 & $(24-31)$ & 26 & $(24-31)$ & 27 & $(26-33)$ & 26 & $(24-31)$ \\
\hline Chronic Disease PRIOR Covid-19 - no. (\%) & $31 / 43$ & $(72.1)$ & $73 / 84$ & $(86.9)$ & $12 / 18$ & $(66.7)$ & $85 / 102$ & $(83.3)$ \\
\hline Chronic lung disease - no. (\%) & $13 / 43$ & $(30.2)$ & $15 / 84$ & $(17.9)$ & $5 / 18$ & $(27.8)$ & $20 / 102$ & $(19.6)$ \\
\hline Chronic kidney disease - no. (\%) & $4 / 43$ & $(9.3)$ & $16 / 84$ & $(19)$ & $0 / 18$ & (0) & $16 / 102$ & $(15.7)$ \\
\hline Renal replacement therapy- no. (\%) & $1 / 43$ & (2.3) & $4 / 84$ & $(4.8)$ & $0 / 18$ & $(0)$ & $4 / 102$ & (3.9) \\
\hline Malignant tumor disease - no. (\%) & $8 / 43$ & $(18.6)$ & $12 / 84$ & $(14.3)$ & $1 / 18$ & $(5.6)$ & $13 / 102$ & $(12.7)$ \\
\hline Diabetes mellitus - no. (\%) & $4 / 43$ & $(9.3)$ & $23 / 84$ & $(27.4)$ & $6 / 18$ & (33.3) & $29 / 102$ & $(28.4)$ \\
\hline Immunosuppressive therapy - no. (\%) & $13 / 43$ & $(30.2)$ & $22 / 84$ & $(26.2)$ & $3 / 18$ & (33.3) & $25 / 102$ & $(24.5)$ \\
\hline
\end{tabular}

Table 1. Baseline Characteristics (urine-status on admission to hospital)

Patients' characteristics of individuals

$\mathrm{IQR}=$ interquartile range

$\mathrm{BMI}=$ Body Mass Index 


\begin{tabular}{|c|c|c|c|c|c|c|c|c|}
\hline \multirow{2}{*}{$\begin{array}{l}\text { Outcome } \\
\text { Primary endpoint }\end{array}$} & \multicolumn{2}{|c|}{ green $(\mathrm{N}=43)$} & \multicolumn{2}{|c|}{ yellow $(\mathrm{N}=84)$} & \multicolumn{2}{|c|}{$\operatorname{red}(\mathrm{N}=18)$} & \multicolumn{2}{|c|}{ non-green $(\mathrm{N}=102)$} \\
\hline & & & & & & & & \\
\hline ICU or Death until day $10-$ no. (\%) & $10 / 43$ & $(23.3)$ & $43 / 84$ & $(51.2)$ & $18 / 18$ & $(100.0)$ & $61 / 102$ & $(59.8)$ \\
\hline \multicolumn{9}{|l|}{ Secondary endpoints - Complications } \\
\hline Death during hospital stay - no. (\%) & $5 / 43$ & $(11.6)$ & $23 / 84$ & $(27.4)$ & $7 / 18$ & $(38.8)$ & $30 / 102$ & $(29.4)$ \\
\hline Still at risk of Death in hospital - no. (\%) & $1 / 43$ & $(2.3)$ & $8 / 84$ & $(9.5)$ & $3 / 18$ & $(16.7)$ & $11 / 102$ & $(10.8)$ \\
\hline ICU level 3 during hospital stay - no. (\%) & $9 / 43$ & $(20.9)$ & $38 / 84$ & $(45.2)$ & $17 / 18$ & $(94.4)$ & $55 / 102$ & $(53.9)$ \\
\hline Mechanical ventilation - no. (\%) & $6 / 43$ & $(14)$ & $28 / 84$ & $(33.3)$ & $17 / 18$ & $(94.4)$ & $45 / 102$ & $(44.1)$ \\
\hline ECMO therapy - no. (\%) & $1 / 43$ & $(2.3)$ & $5 / 84$ & $(6.0)$ & $6 / 18$ & $(33.3)$ & $11 / 102$ & $(10.8)$ \\
\hline Renal replacement therapy $\mathbf{R R T}$ - no. (\%) & $5 / 43$ & $(11.6)$ & $20 / 83$ & $(24.1)$ & $11 / 18$ & $(61.1)$ & $31 / 101$ & $(30.7)$ \\
\hline \multicolumn{9}{|l|}{ Secondary endpoints - Resources } \\
\hline Time on mechanical ventilation - days (DPP) & 23 & $(2.6)$ & 23 & $(7.7)$ & 10 & $(9.4)$ & 19 & $(8.4)$ \\
\hline Time on ECMO therapy - days (DPP) & 11 & $(0.3)$ & 20 & $(1.2)$ & 23 & $(7.7)$ & 22 & (2.3) \\
\hline Time on RRT - days (DPP) & 23 & $(2.7)$ & 17 & $(4.1)$ & 13 & $(7.9)$ & 15 & $(4.6)$ \\
\hline \multicolumn{9}{|l|}{ Secondary endpoints - Blood values } \\
\hline \multicolumn{9}{|l|}{ Median serum-albumin (IQR) - g/dl } \\
\hline first value in hospital (IQR) - g/dl & 3.3 & $(0.9)$ & 2.8 & $(0.9)$ & 1.9 & $(0.7)$ & 2.7 & (1) \\
\hline lowest value (IQR) - g/dl & 3.1 & $(0.7)$ & 2.2 & $(1.1)$ & 1.5 & $(0.4)$ & 2.1 & (1.1) \\
\hline$<2.0 \mathrm{~g} / \mathrm{dl}$ during hospital stay - no. (\%) & $1 / 33$ & $(3.3)$ & $21 / 70$ & $(30)$ & $17 / 18$ & $(94.4)$ & $38 / 88$ & $(43.2)$ \\
\hline \multicolumn{9}{|l|}{ Median antithrombin III (IQR) - \% } \\
\hline first value in hospital (IQR) - \% & 95 & $(23.5)$ & 93 & $(22)$ & 68 & (34) & 89 & $(24.5)$ \\
\hline lowest value (IQR) - \% & 95 & (26) & 78 & $(40)$ & 52.5 & (43) & 71 & (42) \\
\hline$<70 \%$ during hospital stay - no. (\%) & $5 / 22$ & $(22.7)$ & $21 / 52$ & $(40.4)$ & $12 / 18$ & $(66.7)$ & $33 / 70$ & $(47.1)$ \\
\hline
\end{tabular}

Table 2. Primary and secondary outcome measures (urine-status on admission to hospital)

Patients' outcome measures including the primary endpoint and secondary endpoints.

$\mathrm{IQR}=$ interquartile range

ICU=Intensive Care Unit (level 3)

$\mathrm{ECMO}=$ Extra-corporal membrane oxygenation

$\mathrm{RRT}=$ Renal Replacement Therapy

$\mathrm{DPP}=$ Days Per Patient (estimate of patient days on mechanical ventilation, ECMO or RRT per patient in each group) 


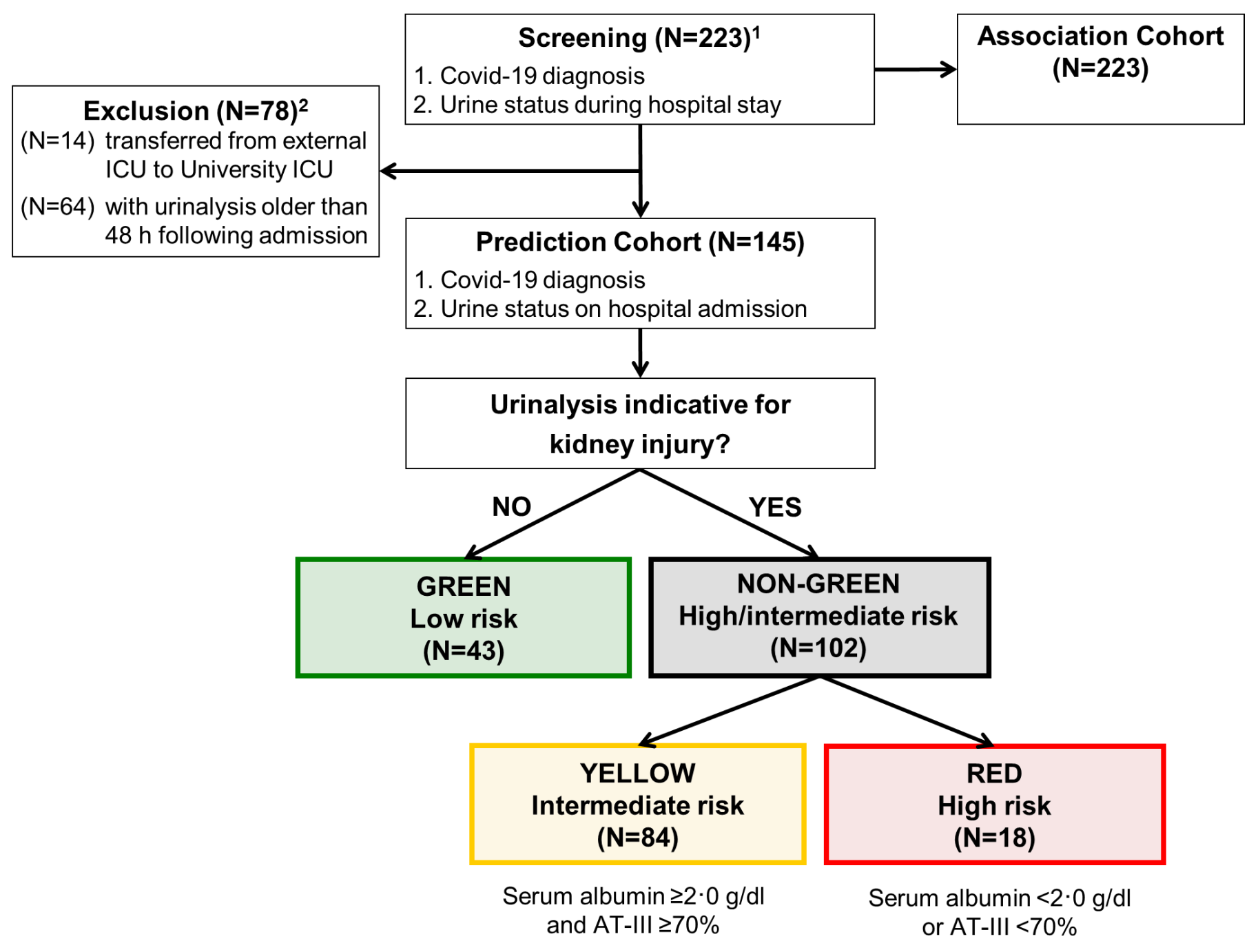

Figure 1. Algorithm for Prediction Model Development (Study Flow Chart)

Patients with urinary status on admission to hospital were allocated to low, intermediate and high-risk.

The results of the prediction analysis are shown in Tables 1, 2 and Figures 2, 3. The first event within 10 days was counted, admission to ICU or death, whatever came first.

${ }^{1}$ Patients, who initially served for generation of the algorithm were not part of this cohort. ${ }^{19}$

${ }^{2}$ Patients, who were transferred from external ICU to University ICU $(\mathrm{N}=14)$ and patients with urinary status older than $48 \mathrm{~h}$ following admission $(\mathrm{N}=64)$ were excluded from prediction analysis and included to the association analysis, which is shown in Supplementary Table 1.

$\mathrm{ICU}=$ Intensive Care Unit

AT-III = Antithrombin III activity 


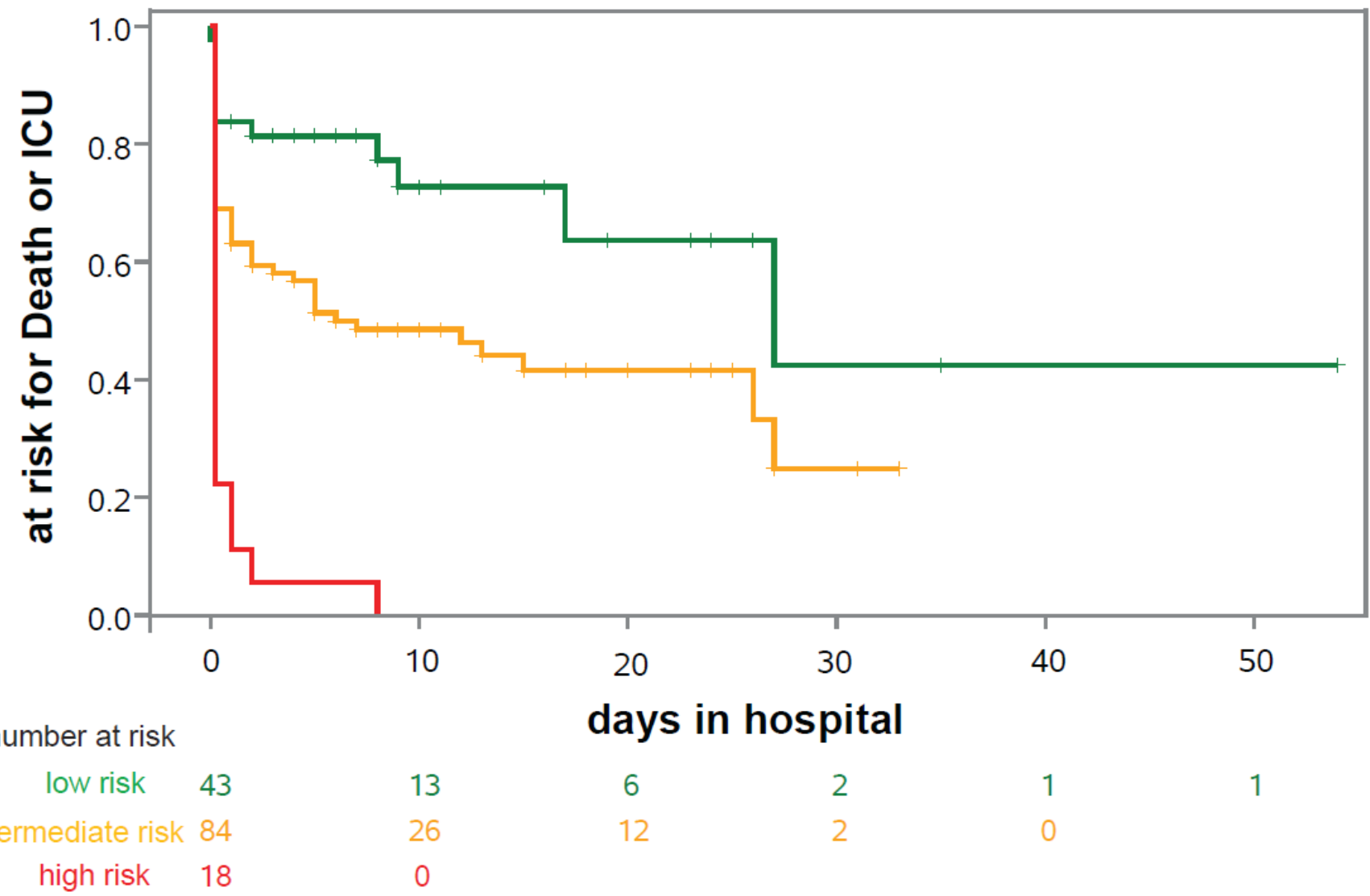

Figure 2. Prediction of "death or need for ICU" on entry to hospital

Kaplan-Meier estimates prediction analysis for patients with urinary status on admission to hospital: patients without kidney injury (low-risk; green; $\mathrm{N}=43$ ) vs. patients with kidney injury, divided in intermediate-risk (yellow; $N=84$ ) and high-risk (red; $\mathrm{N}=18)$. 


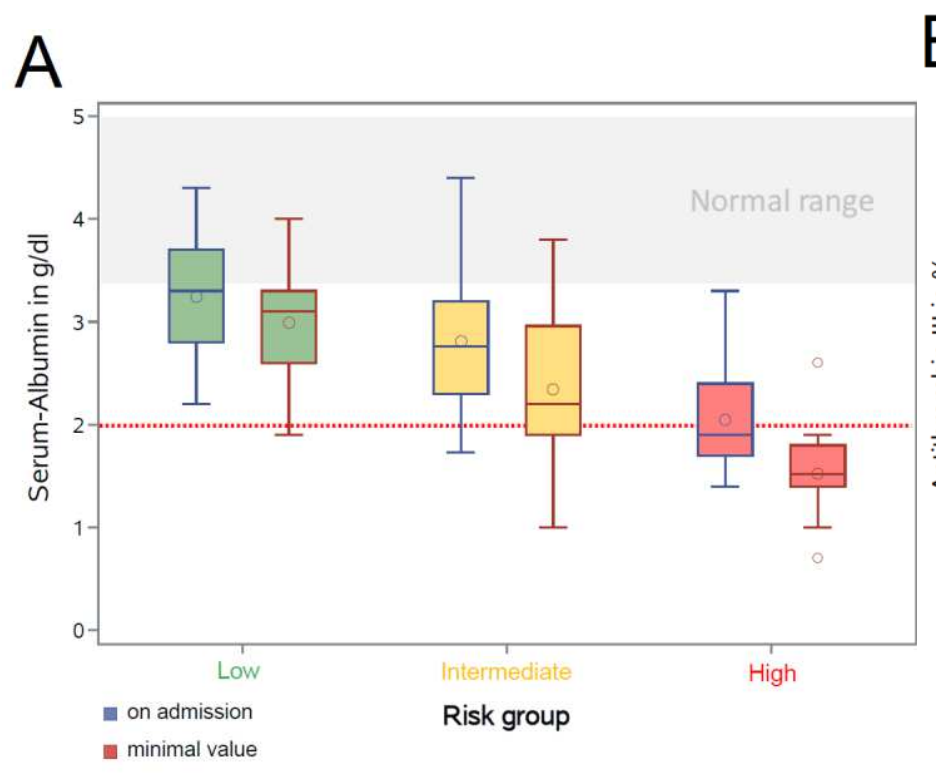

B

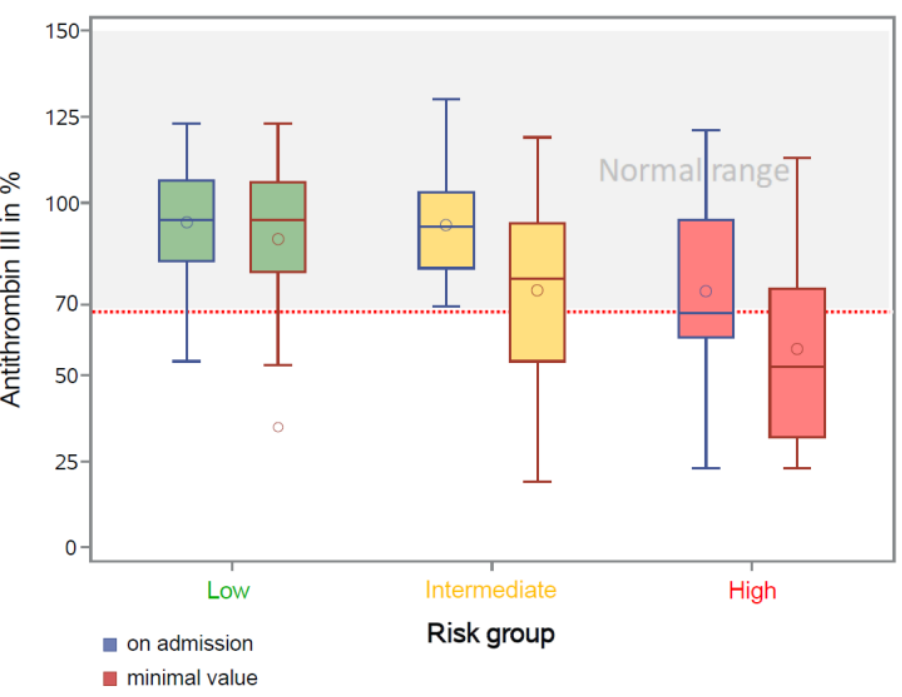

Figure 3. Differences and development of serum-albumin and antithrombin III of the different cohorts (low, intermediate and high risk) within the prediction analysis

Panel A Serum albumin on hospital admission and minimum level during hospital stay.

Panel B Antithrombin III (AT-III) activity on hospital admission and minimum level during hospital stay.

Both, serum-albumin and AT-III illustrate differences in-between low-risk (green) and intermediate or high-risk (yellow and red). Remarkably, a substantial number of patients in the intermediate-risk group, but not the low-risk group, exhibit a worsening course of their hospital stay - reflecting the possible benefits of our algorithm as an early warning system in Covid-19 systemic disease. 


\begin{tabular}{|c|c|c|c|c|c|}
\hline \multirow{3}{*}{\begin{tabular}{|l} 
Characteristic \\
Male sex - no. (\%)
\end{tabular}} & \multicolumn{5}{|c|}{$\begin{array}{l}\text { Association: urine-status in relation to disease severity } \\
\qquad(\mathrm{n}=223)\end{array}$} \\
\hline & \multicolumn{2}{|c|}{ green $(n=58)$} & \multicolumn{2}{|c|}{ non-green $(n=165)$} & \multirow{2}{*}{$\begin{array}{r}\begin{array}{c}\text { Green vs. } \\
\text { non-green }\end{array} \\
0.98\end{array}$} \\
\hline & $40 / 58$ & (69) & $114 / 165$ & $(69.1)$ & \\
\hline Median age (IQR) - year & 60 & $(25)$ & 66 & (19) & $<0.01$ \\
\hline Median body mass index BMI (IQR) & 27.2 & $(7.5)$ & 27.4 & (6.9) & 0.778 \\
\hline Chronic Disease PRIOR Covid-19 - no. (\%) & $44 / 58$ & $(75.9)$ & $122 / 165$ & $(73.9)$ & \\
\hline Chronic heart disease - no. (\%) & $16 / 58$ & $(27.6)$ & $46 / 165$ & $(27.9)$ & \\
\hline Chronic lung disease - no. (\%) & $16 / 58$ & $(27.6)$ & $28 / 165$ & $(17)$ & \\
\hline Chronic kidney disease - no. (\%) & $6 / 58$ & $(10.3)$ & $17 / 165$ & $(10.3)$ & \\
\hline Renal replacement therapy- no. (\%) & $1 / 58$ & $(1.7)$ & $4 / 165$ & $(2.4)$ & \\
\hline Malignant tumor disease - no. (\%) & $13 / 58$ & $(22.4)$ & $14 / 165$ & (8.5) & \\
\hline Diabetes mellitus - no. (\%) & $8 / 58$ & $(13.8)$ & $35 / 165$ & $(21.2)$ & \\
\hline Immunosuppressive therapy - no. (\%) & $19 / 58$ & $(32.8)$ & $29 / 165$ & $(17.6)$ & \\
\hline \multicolumn{6}{|l|}{ Median serum-albumin (IQR) - g/dl } \\
\hline first value in hospital (IQR) - g/dl & 3.3 & $(0.9)$ & 2.4 & $(0.9)$ & \\
\hline lowest value (IQR) - g/dl & 3 & $(0.9)$ & 2 & $(0.8)$ & \\
\hline$<2.0 \mathrm{~g} / \mathrm{dl}$ during hospital stay - no. (\%) & $3 / 47$ & $(6.4)$ & $70 / 148$ & $(47.3)$ & \\
\hline \multicolumn{6}{|l|}{ Median antithrombin III (IQR) - \% } \\
\hline first value in hospital (IQR) - \% & 92.5 & (23) & 85 & 28 & \\
\hline lowest value (IQR) - \% & 90 & (30) & 63 & 37 & \\
\hline$<70 \%$ during hospital stay - no. (\%) & $7 / 28$ & (25) & $67 / 118$ & $(56.8)$ & \\
\hline ICU or Death until day $10-$ no. (\%) & $14 / 58$ & $(24.1)$ & $109 / 164$ & $(66.5)$ & $<0.01$ \\
\hline Death during hospital stay - no. (\%) & $7 / 58$ & $(12.1)$ & $47 / 165$ & $(28.5)$ & $<0.01$ \\
\hline Still at risk of Death in hospital - no. (\%) & $2 / 58$ & (3.4) & $30 / 165$ & $(18.2)$ & \\
\hline ICU level 3 during hospital stay - no. (\%) & $13 / 58$ & $(22.4)$ & $106 / 165$ & $(64.2)$ & \\
\hline Mechanical ventilation - no. (\%) & $9 / 58$ & $(15.5)$ & $95 / 165$ & $(57.6)$ & $<0.01$ \\
\hline ECMO therapy - no. (\%) & $1 / 58$ & $(1.7)$ & $31 / 164$ & $(18.9)$ & $<0.01$ \\
\hline Renal replacement therapy RRT - no. (\%) & $7 / 58$ & $(12.1)$ & $65 / 164$ & $(39.6)$ & $<0.01$ \\
\hline
\end{tabular}

\section{Supplemental Table 1 Association (all patients with urine-status)}

Patients' characteristics of individuals with urine-status including the primary endpoint and secondary endpoints.

$\mathrm{ICR}=$ interquartile range

ICU=Intensive Care Unit (level 3)

$\mathrm{ECMO}=$ Extra-corporal membrane oxygenation

$\mathrm{RRT}=$ Renal Replacement Therapy

$\mathrm{BMI}=$ Body Mass Index 


\begin{tabular}{|l|r|r|r|c|}
\hline Parameter & Hazard Ratio & 95\% confidence intervals & \multicolumn{1}{|c|}{$\mathbf{P}$} \\
\hline Kidney injury & $\mathbf{2 . 8 6 8}$ & $\mathbf{1 . 4 8 4}$ & $\mathbf{5 . 5 0 5}$ & $\mathbf{0 . 0 0 1 7}$ \\
\hline Age [year] & 0.995 & 0.979 & 1.011 & 0.5412 \\
\hline Sex: male & 1.088 & 0.660 & 1.794 & 0.7417 \\
\hline Chronic heart disease & 1.191 & 0.673 & 2.110 & 0.5480 \\
\hline Chronic lung disease & 1.311 & 0.756 & 2.273 & 0.3355 \\
\hline Chronic kidney disease & 0.645 & 0.275 & 1.513 & 0.3138 \\
\hline RRT & 3.132 & 0.897 & 10.938 & 0.0736 \\
\hline Malignant tumor disease & 0.888 & 0.337 & 2.344 & 0.8109 \\
\hline Diabetes mellitus & 0.973 & 0.557 & 1.701 & 0.9247 \\
\hline Immunosuppressive therapy & 0.938 & 0.444 & 1.980 & 0.8660 \\
\hline
\end{tabular}

Supplemental Table 2 Adjustment of the Covid-19 kidney injury prediction model for age, sex and co-morbidities

Adjustment revealed in a robust hazard ratio for Covid-19 kidney injury, which was consistent with the unadjusted hazard ratio. Only 5 patients were on chronic RRT prior Covid-19 infection, resulting in a broad range in the $95 \%$ confidence interval and a hazard ration of 3.1 , which just failed to reach the level of significance.

RRT=Renal Replacement Therapy 


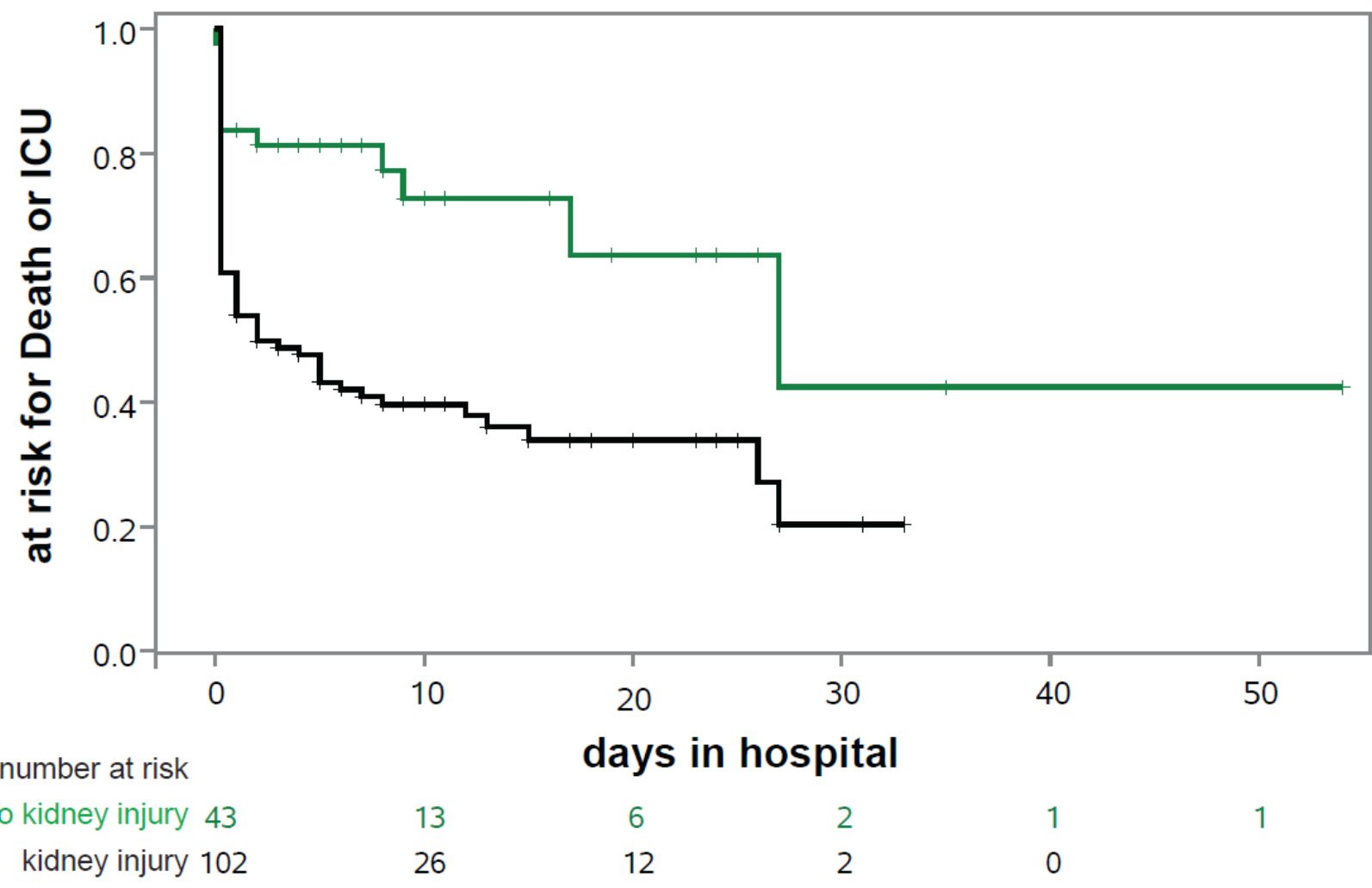

Supplemental Figure 1. Prediction of "death or need for ICU" on entry to hospital Kaplan-Meier estimates prediction analysis for patients with kidney injury (non-green; $n=102$ ) vs. patients without kidney injury (normal urine-status; green; $n=43$ ). 


\section{Figures}

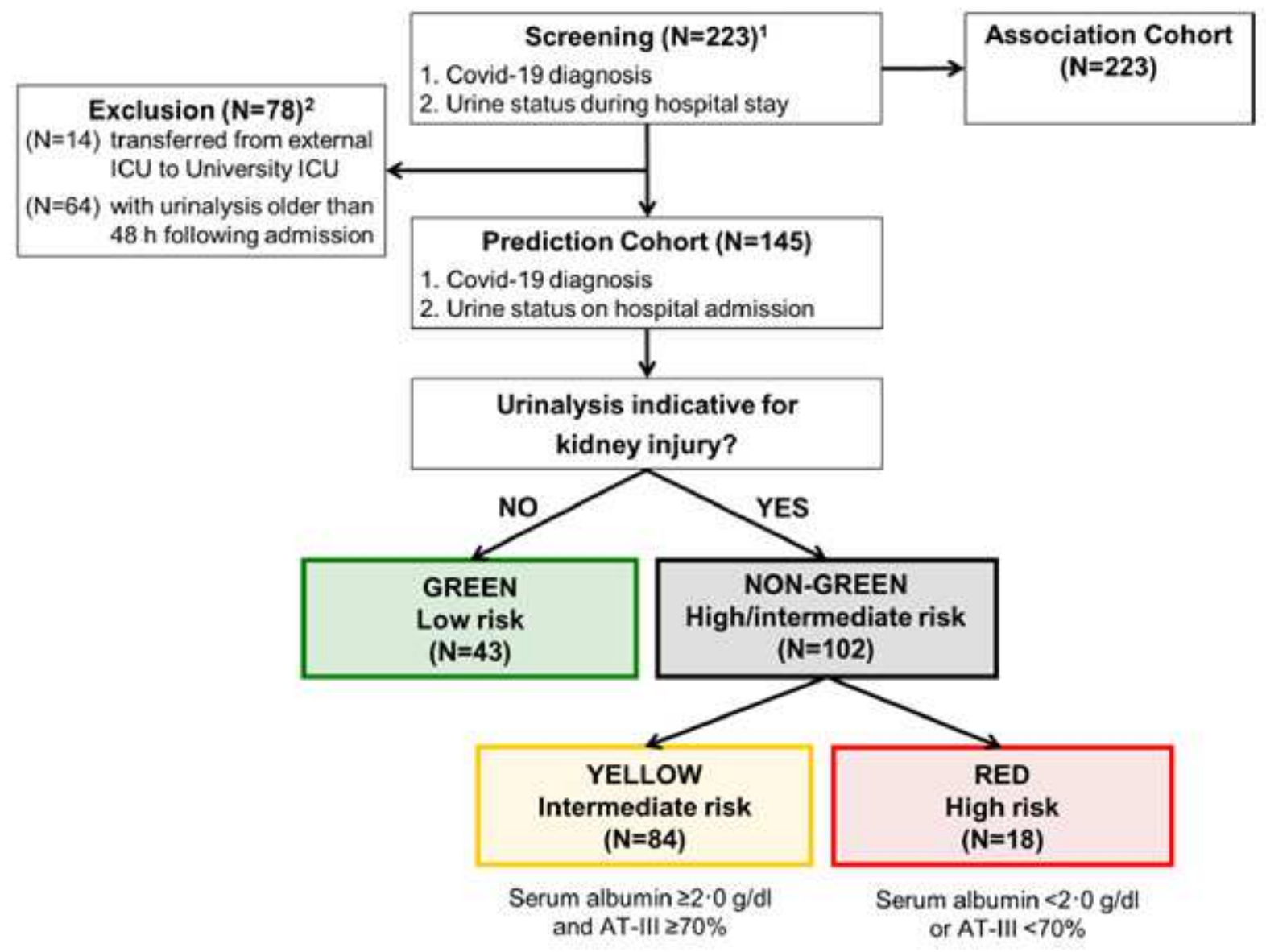

Figure 1

Algorithm for Prediction Model Development (Study Flow Chart) Patients with urinary status on admission to hospital were allocated to low, intermediate and high-risk. The results of the prediction analysis are shown in Tables 1, 2 and Figures 2, 3. The first event within 10 days was counted, admission to ICU or death, whatever came first. 1 Patients, who initially served for generation of the algorithm were not part of this cohort.19 2 Patients, who were transferred from external ICU to University ICU (N=14) and patients with urinary status older than $48 \mathrm{~h}$ following admission $(\mathrm{N}=64)$ were excluded from prediction analysis and included to the association analysis, which is shown in Supplementary Table 1. ICU = Intensive Care Unit AT-III = Antithrombin III activity 


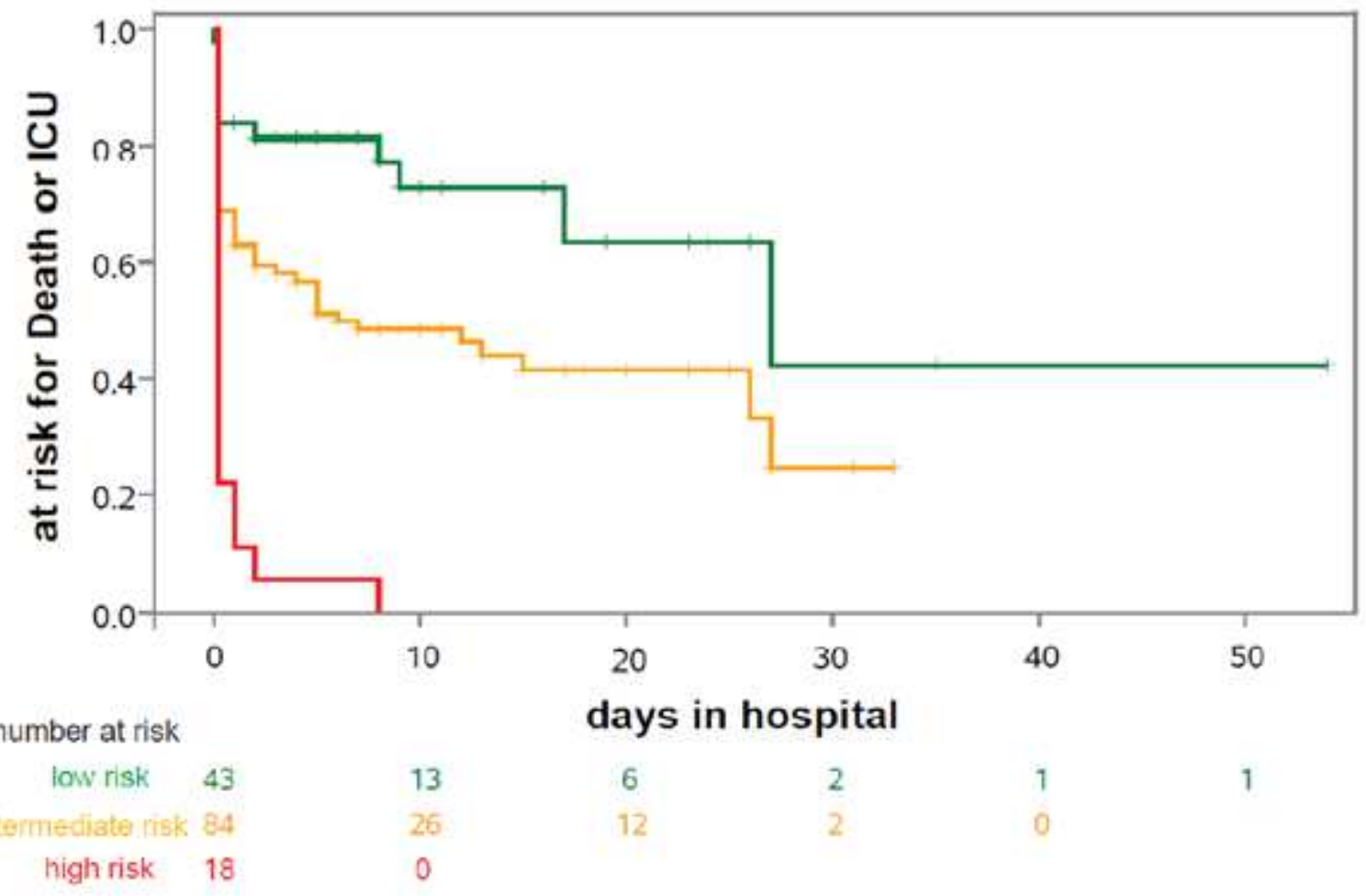

Figure 2

Prediction of "death or need for ICU" on entry to hospital Kaplan-Meier estimates prediction analysis for patients with urinary status on admission to hospital: patients without kidney injury (low-risk; green; $\mathrm{N}=43$ ) vs. patients with kidney injury, divided in intermediate-risk (yellow; $\mathrm{N}=84$ ) and high-risk (red; $\mathrm{N}=18$ ).
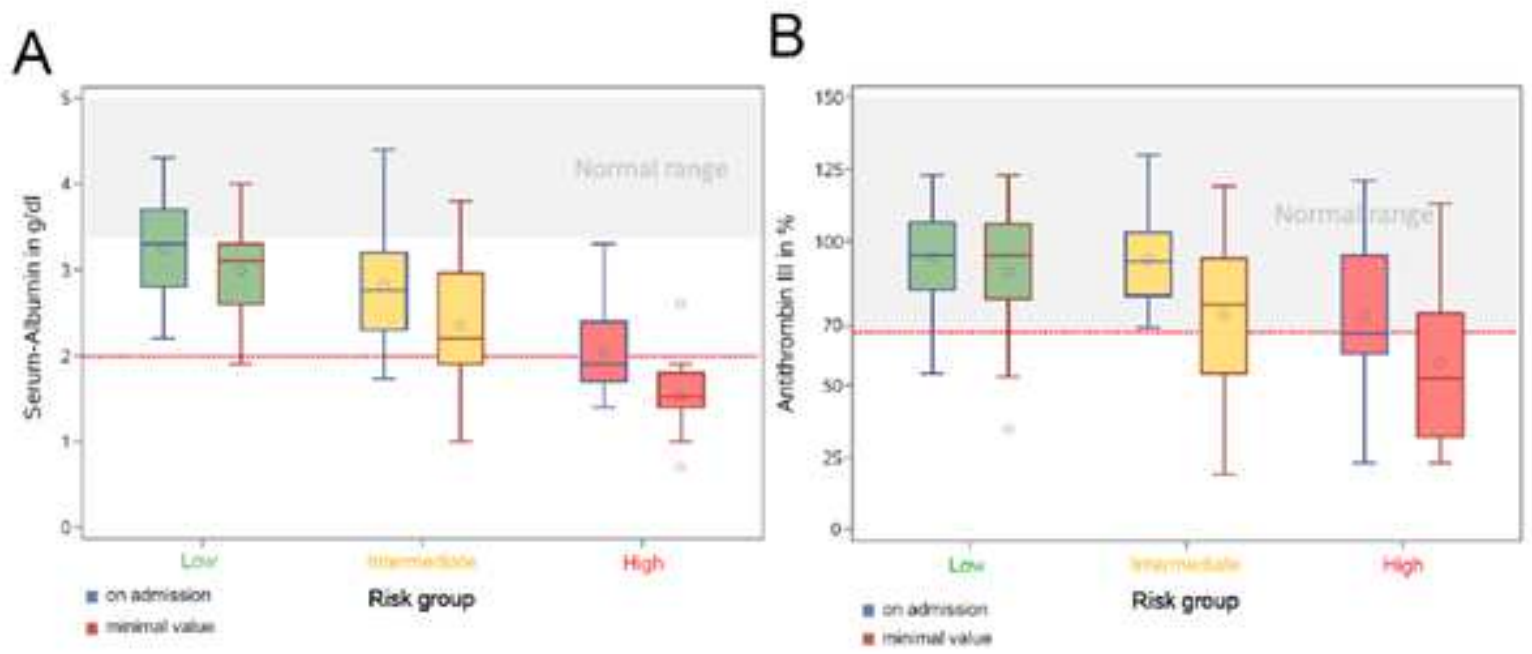

Figure 3

Differences and development of serum-albumin and antithrombin III of the different cohorts (low, intermediate and high risk) within the prediction analysis Panel A Serum albumin on hospital admission 
and minimum level during hospital stay. Panel B Antithrombin III (AT-III) activity on hospital admission and minimum level during hospital stay. Both, serum-albumin and AT-III illustrate differences in-between low-risk (green) and intermediate or high-risk (yellow and red). Remarkably, a substantial number of patients in the intermediate-risk group, but not the low-risk group, exhibit a worsening course of their hospital stay - reflecting the possible benefits of our algorithm as an early warning system in Covid-19 systemic disease. 